\title{
Pilot Placement Schemes for Channel Estimation of Proposed 5G-GFDM System
}

\author{
Suleman Tahir ${ }^{1, *}$, Shahzad Amin Sheikh ${ }^{1}$, and Omer Bin Saeed ${ }^{2}$ \\ ${ }^{1}$ Department of Electrical Engineering, College of Electrical and Mechanical Engineering, NUST, Islamabad, Pakistan \\ ${ }^{2}$ Department of Computer Engineering, College of Electrical and Mechanical Engineering, NUST, Islamabad, Pakistan
}

\begin{abstract}
Orthogonal Frequency Division Multiplexing (OFDM) is a highly regarded technique used in the $4 \mathrm{G}$ mobile communication systems to provide reliable communication and high data rates due to the orthogonality between its sub carriers. However, it cannot be used in the next generation cellular system i.e. 5G. Thus, a new technique Generalized Frequency Division Multiplexing (GFDM) has been proposed to meet the demands of the next generation systems, which are higher data rates than $4 \mathrm{G}$, minimum response time, lower power consumption etc. GFDM is a non-orthogonal, multicarrier scheme, which seems to fulfil the requirements of the new wireless communication system. The aim of this paper is to use the pilot symbols and their optimum placements within the data for the channel estimation of the GFDM system. It is shown that the optimum arrangement of the pilot symbols is to place them uniformly on equal intervals within the data and to cluster them in the middle of the data.
\end{abstract}

\section{INTRODUCTION}

Cellular communication has become one of the most essential needs of modern days. First generation $1 \mathrm{G}$ of the mobile communication introduced the world to voice transmission, which is very basic yet innovative. Considering the needs, a second-generation communication system was introduced which improved the battery performance of the devices and digitalized the voice thus increasing the system efficiency and capacity and quality of service. $2 \mathrm{G}$ system also revolutionized the world by the introduction of a service of text message. The data rates and the internet speed were then matched to the wired systems by the introduction of $3 \mathrm{G}$ transmission system. 3G brought a new era of smart phones, HD screens, large and expandable storage, higher processing capability, state of the art cameras etc. It also introduced the social media, which made the users demand even higher data rates, and system reliability, which set forth the world of fourth generation (4G), and LTE [1].

Wireless communication shifted from single carrier to multicarrier transmission due to its advantages of high data rates, reliable communication etc. in the multipath fading channels. In $4 \mathrm{G}$ and LTE orthogonal frequency division multiplexing proven itself to be a state of the art system of communication. It introduced the concept of orthogonality between the subcarriers, which is one of the main reasons of such high data rates. However, it becomes impossible for OFDM to address the new requirements of the communication system of the next generation. The requirements of the modern communication system are beyond data capacity and throughput of the OFDM systems. Applications of low latency in tactile internet [2], sporadic traffic and dynamic spectrum access for cognitive radio and coarse synchronization motivated the experts and scientists to augment their research on other more efficient technique, which cannot just match the previous one but also enhance it to the next level. The main drive to introduce and to design such a technique is machine type communication, internet of things and application that in turn will bring the low-cost sensors in the $5 \mathrm{G}$ systems and communication will be able to carry out with rough synchronization (asynchronization). Generalized Frequency Division Multiplexing (GFDM), a well-known non-orthogonal multiplexing technique is being researched widely to meet the needs of the new era. MIMO-GFDM communication systems by fully utilizing the frequency diversity can outperform the MIMOOFDM systems [3], [4] and [5]. As in OFDM, cyclic prefix (CP) is also used in GFDM to minimize the intersymbol interference. GFDM is also being considered for the cognitive radio application due to its low out of band radiation (OOB) [6]. However, a self-induced interference emerged in GFDM due to its non-orthogonal nature, which is because of the pulse shaping subcarriers in GFDM system. Therefore, the focus was to design such a receiver, which can cancel this interference effect. This was the reason that only AWGN channels were being used for the research purposes. However now the multipath fading channels are also being considered as in [7] and [8]. Recently for GFDM, the focus is channel equalization and estimation. Different schemes and techniques are being introduced to cater this problem. As in OFDM, pilot-based channel estimation is widely being researched

\footnotetext{
*Corresponding author: p116006@nu.edu.pk
} 
in GFDM. Due to the frequency selectivity in broadband communication, a more complex and sophisticated scheme of pilot insertion and channel estimation algorithms need to be implemented for more efficient channel equalization and estimation. Training based estimation is one of the most effective channel estimation technique. Known symbols along with the data are sent to study the channel response, which is then reverse engineered for channel estimation. The optimum pilot design and placement has been widely researched by many [9]-[11]. In [12] the optimum pilot tone selection for the orthogonal frequency division multiplexing (OFDM) is considered which minimizes the MSE of the minimum MSE (MMSE).

In this article, optimum pilot placement scheme is considered for the channel estimation for GFDM systems. In [13] it is suggested that placement technique that gives the optimum results is that the higher power pilot symbols should be placed in the center of the pilot packet and the pilot symbols arranged uniformly on equal intervals within the data. This placement technique along with the other i.e. the pilot symbols clustered in the center of the data are applied on the (GFDM) to enhance the channel estimation for the GFDM.

TABLE 1: Table of Notations

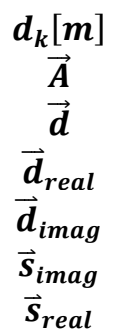

Pulse shaped data Matrix form of A

Data vector

Real part of the data

Imaginary part of the data

Imaginary part of $\overrightarrow{\boldsymbol{s}}$

Real part of $\overrightarrow{\boldsymbol{s}}$

\section{GENERALIZED FREQUENCY DIVISION MULTIPLEXING}

\section{TRANSMITTER}

Fig. 1 illustrates a block diagram of the transmitter of generalized frequency division multiplexing (GFDM) system. Let $\vec{a}$ is a resultant vector after $\mathrm{A} / \mathrm{D}$ convertor. Then a mapper i.e. QPSK (quadrature phase shift keying) is used which converts the data to symbols of complex constellation $\vec{q}$. A raised cosine filter then pulse shapes the QPSK modulated data. The resulting vector $\overrightarrow{q_{c}}$ contains $\mathrm{N}$ symbols which (for GFDM) can be decomposed into $\mathrm{M}$ sub-symbols and $\mathrm{K}$ subcarriers and the total number of symbols are defined as $N=K M$. The entries of data matrix $\boldsymbol{A}$ of the GFDM belongs to the complex numbers and is of order $K \times M$, where $\mathrm{K}$ and $\mathrm{M}$ is the total number of subcarriers and sub-symbols respectively. $d_{k}[m]$ represents the sub-symbols and subcarriers at time $m$ and index $\mathrm{k}$ respectively. Each entry of $d_{k}[m]$ is pulse shaped. The GFDM transmitter [8] is given as under:

$$
s[n]=\sum_{k=0}^{K-1} \sum_{m=0}^{M-1} d_{k}[m] g_{t x_{k, m}}[n]
$$

Where

$$
g_{t x_{k, m}}[n]=g_{t x}[(n-m k) \bmod N] e^{-j \frac{2 \pi k n}{K}}
$$

And $n=\{0,1,2, \cdots, N-1\}, N=M K$. Modulo operation in the above equation is responsible for the circular filtering. In matrix form, the above equation can be written as:

$\vec{S}=$

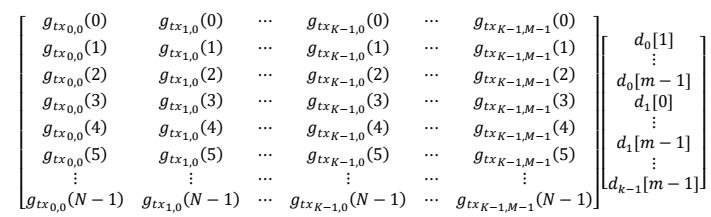

$g_{t x_{0,0}}(N-1) \quad g_{t x_{10}}(N-1)$

$$
\begin{array}{ll} 
& \overrightarrow{\boldsymbol{s}}=\overrightarrow{\boldsymbol{A}} \cdot \overrightarrow{\boldsymbol{d}} \\
\text { i.e. } & \overrightarrow{\boldsymbol{d}}=d_{K}[m] \\
\text { As } & \overrightarrow{\boldsymbol{d}}=\vec{d}_{\text {real }}+\vec{d}_{\text {imaginary }}
\end{array}
$$

It means that $\overrightarrow{\boldsymbol{d}}$ contains all the complex numbers. Thus, it is to be kept in mind that $\overrightarrow{\boldsymbol{A}}$ is to be calculated separately for both real and imaginary parts of $\overrightarrow{\boldsymbol{d}}$. Then $\vec{s}$ will be calculated separately for both parts of $\overrightarrow{\boldsymbol{d}}$ and then these parts are added together as:

$$
\vec{s}=\overrightarrow{\boldsymbol{s}}_{\text {real }}+\overrightarrow{\boldsymbol{s}}_{\text {imag }}
$$

Generalized frequency division multiplexing is a nonorthogonal waveform, which implements the approach of classical filter band digitally. Cyclic prefix (as in OFDM) is also used in GFDM, which allows the implementation of lower complexity equalization system at the receiver. Cyclic prefix is implemented in such a way that the last some bits of the next data block are put between the data block in order to avoid the high cost and more complexed receivers. To increase the spectral efficiency, cyclic prefix is shortened using the tail biting technique.

\subsection{PILOT SYMBOL PLACEMENT}

It is assumed that each data packet is denoted by $d_{b}=$ $\left\{d_{b}[1], d_{b}[2], d_{b}[3], \ldots, d_{b}[N]\right\}$ consisting of $N$ data symbols and $p_{b}=\left\{p_{b}[1], p_{b}[2], p_{b}[3], \ldots, p_{b}[N]\right\}$ i.e. $\mathrm{P}$ pilot symbols.

Figure 2 illustrates the first scenario of the arrangement of pilot symbols within the data packet. Pilot symbols are arranged uniformly at equal intervals within the packet.

Fig. 3 shows the second method covered in this paper in which the pilots symbols are clustered in the center of the data.

\footnotetext{
*Corresponding author: p116006@nu.edu.pk
} 


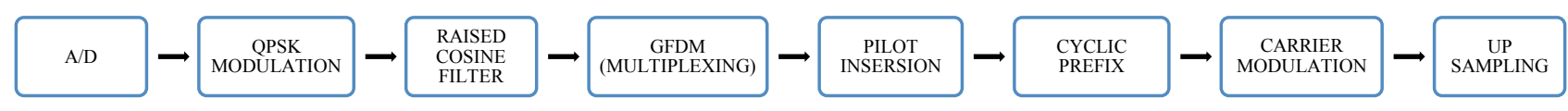

Fig. 1. GFDM Transmitter

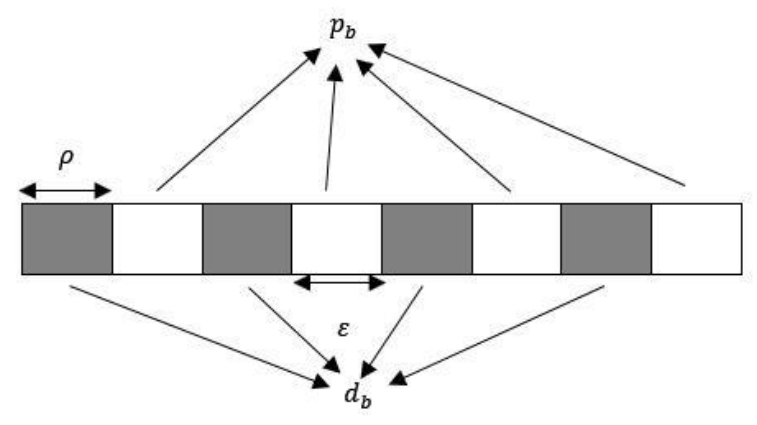

Fig. 2. Arrangement of Pilot Symbols uniformly in the data packet (Method I)

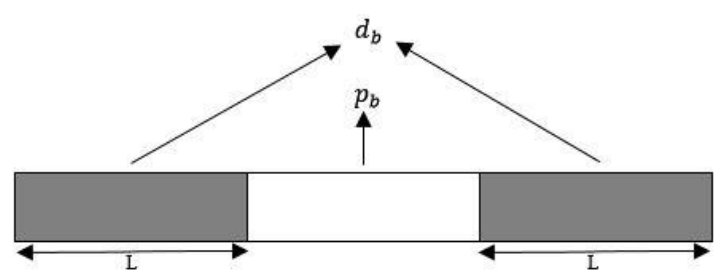

Fig. 3. Pilot Symbols are clustered in the center of data packet (Method 2)

Let the data block length vector be $\boldsymbol{\rho}=$ $\left[\rho_{1}, \rho_{2}, \rho_{3}, \ldots, \rho_{n+1}\right]$ and $\varepsilon=\left[\varepsilon_{1}, \varepsilon_{2}, \varepsilon_{3}, \ldots, \varepsilon_{n+1}\right]$ be the vector of pilot cluster length, then the pilot symbol placement of $\mathrm{n}$ clusters can be generally represented by $\mathbb{P}=(\boldsymbol{\rho}, \boldsymbol{\varepsilon})$. Considering fig. 1 , if the pilot symbol is placed in the start then $\rho_{1}=0$ and for the placements which ends with the pilot symbols $\rho_{n+1}=0$. There will be $\sum_{i=0}^{n} \rho_{i}=N$ and $\sum_{i=0}^{n} \varepsilon_{i}=P$ data and pilot symbols respectively.

Now for the second scenario as in fig. 3, the pilot symbols are clustered in the center of the data. The position of the pilot symbols and the data symbols in one packet have also been illustrated i.e. data symbols are placed in the first and last $\mathrm{L}$ position of the packet and the rest of the parts $[L+1, N+P-L]$ are the pilot symbols.

The only parts of the packet corresponding to the pilot symbols are to be used for the training-based channel estimation. Mathematically the input symbol can be decomposed into two parts:

$$
\overrightarrow{\boldsymbol{s}}=\overrightarrow{\boldsymbol{d}}_{\boldsymbol{b}}+\overrightarrow{\boldsymbol{p}}_{\boldsymbol{b}}
$$

Where $\overrightarrow{\boldsymbol{d}}_{\boldsymbol{b}}$ represents the vector containing the data vector and $\overrightarrow{\boldsymbol{p}}_{\boldsymbol{b}}$ represents the pilot symbols. And $d_{\boldsymbol{b}}$ can be obtained by setting the pilot symbol vector zero.
Considering the pilot symbol placement as illustrated in fig. 1. $\mathbb{P}=(\boldsymbol{\rho}, \boldsymbol{\varepsilon})$ represents the placement of pilot symbols within the data. However, for the pilot symbols all placed in the center of the data, shift invariant property of Cramer Rao Bound (CRB) is concluded by a lemma in [13]:

$$
\Lambda\left(\rho, \varepsilon, p_{b}\right)=\Lambda\left(\rho+e_{1}-e_{n+1}, \varepsilon, p_{b}\right)
$$

Where $\boldsymbol{e}_{\boldsymbol{i}}$ is a single row vector with zeros except the $i$ th position which contains one. In communication system, most of the pilots symbols are designed keeping in mind the constraint modulus $(\mathrm{CM})$ property. i.e. $\left[p_{p}[i]\right]^{2}=\sigma_{p}^{2}$, where $i=1,2,3, \ldots, N+1$ and $\sigma_{p}^{2}$ is the variance of the respective pilot symbol. This constraint is also considered in this article. Two or three zeros are appended on each side of the pilot symbols which gives the lowest CRB [13]. And also, another method is putting two of the data symbols each of length $L$ in the start and at the end of the packet and this also gives the lowest CRB. The total length of the pilot symbols in this case will be N+P-2L. These points are assumed for the optimal placement and design of the pilot symbols: Data symbols and channel taps belong to the i.i.d sequence with zero mean and the channel response $h$, AWGN noise $w$ and the data are jointly independent.

For the pilot symbol power $\sigma_{p}^{2}$ and data symbol power $\sigma_{d}^{2}$, the optimal pilot design and placement needs to satisfy the following [13]:

$$
\begin{aligned}
& \mathbb{P}_{*} \in\left[\left(\boldsymbol{\rho}, \boldsymbol{\varepsilon}: \boldsymbol{\rho}_{1}, \boldsymbol{\rho}_{n+1} \geq L\right)\right] \\
& \left.p_{b_{*}} \in\left[p_{b}: R_{p_{b}}=P \sigma_{p}^{2} \boldsymbol{I}\right)\right]
\end{aligned}
$$

For $\sigma_{p}^{2}=\sigma_{d}^{2}$, the optimal pilot design and placement needs to satisfy the above conditions which I considered in the optimal pilot placement and design for the GFDM communication system.

\subsection{CARRIER MODULATION}

Carrier modulation is then performed just before transmitting the signal i.e. as the last step of the transmitter. The message signal is superimposed over a high frequency cosine wave given as under:

$$
\mathrm{s}=\sqrt{2}\left(\overrightarrow{\boldsymbol{s}}_{\text {real }} \cos \left(2 \pi f_{c} t\right)-\overrightarrow{\boldsymbol{s}}_{\text {imag }} \sin \left(2 \pi f_{c} t\right)\right)
$$

where $f_{c}$ is the carrier frequency.

\footnotetext{
*Corresponding author: p116006@nu.edu.pk
} 


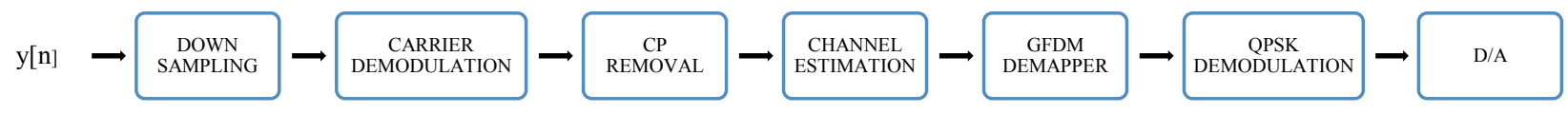

Fig. 4. GFDM Receiver

\section{GFDM RECEIVER}

A frequency block-fading channel is used in this paper with the assumption that for one data packet the random channel will remain the same and will change for the next packet of data. Rayleigh multipath channel is used in this paper and is simulated by Jake's Model as specified in [7]. The model is based on the summing of sinusoids.

The channel is modeled by a linear system as shown below:

$$
y[n]=s[n] * h[n]+w[n]
$$

where $\boldsymbol{y}[\boldsymbol{n}]$ is the received signal such that the transmitted signal $\boldsymbol{s}[\boldsymbol{n}]$ is passed through the frequency flat fading channel with the response of $\boldsymbol{h}[\boldsymbol{n}]$, “*” representing their convolution and the AWGN channel $\boldsymbol{w}[\boldsymbol{n}]$.

Here we have to cater the AWGN noise and the multipath fading as in $y[n]=s[n] * h[n]+w[n]$. It's easy to get rid of the AWGN, the challenge is to equalize or estimate the channel. In general, pilots are used in this paper which tell about the nature of the channel and thus with the help of these pilot symbols equalization or estimation is carried out. It is one of the most effective way of knowing the nature of the channel. Fig. 4 shows a GFDM receiver. According to the equation $y[n]=s[n] * h[n]+w[n]$, the vector $y$ contains the time samples $y[n]$, which is obtained at the receiver after the channel effect $h[n]$ and the AWGN noise $w[n]$. For the Raleigh multipath channel $h[n]$ is a convolution vector which is constructed from the channel response. The convolution of the transmitted signal to the channel response is made circular by adding cyclic prefix at the transmitter.

\subsection{LS RECEIVER:}

Frequency domain channel equalization is carried out in this paper through least squares method. The noisy/channel affected pilot symbols received at the receiver end are compared to original known pilots and thus used as a training sequence for a filter. In this article, Least Squares (LS) method is used for the estimation of the channel $\widehat{\mathbf{H}}$ in such a way that the following cost function is minimized:

$$
\begin{gathered}
(\widehat{\mathbf{H}})=|| \mathbf{Y}-\mathbf{S} \widehat{\mathbf{H}}||^{2} \\
(\widehat{\mathbf{H}})=(\mathbf{Y}-\mathbf{S} \widehat{\mathbf{H}})^{\mathbf{H}}(\mathbf{Y}-\mathbf{S} \widehat{\mathbf{H}}) \\
(\widehat{\mathbf{H}})=\mathbf{Y}^{\mathbf{H}} \mathbf{Y}-\mathbf{Y}^{\mathbf{H}} \mathbf{S} \widehat{\mathbf{H}}-\widehat{\mathbf{H}}^{\mathbf{H}} \mathbf{S}^{\mathbf{H}} \mathbf{Y}+\widehat{\mathbf{H}}^{\mathbf{H}} \mathbf{S}^{\mathbf{H}} \mathbf{S} \widehat{\mathbf{H}}
\end{gathered}
$$

Taking partial derivation w.r.to $\widehat{\mathbf{H}}$ and setting it to zero:

$$
\begin{gathered}
\frac{\partial J(\widehat{\mathbf{H}})}{\partial \widehat{\mathbf{H}}}=-2\left(\mathbf{S}^{\mathbf{H}} \mathbf{Y}\right)^{*}+2\left(\mathbf{S}^{\mathbf{H}} \mathbf{S} \widehat{\mathbf{H}}\right)^{*}=0 \\
\mathbf{S}^{\mathbf{H}} \mathbf{S} \widehat{\mathbf{H}}=\mathbf{S}^{\mathbf{H}} \mathbf{Y} \\
\widehat{\mathbf{H}}=\left(\mathbf{S}^{\mathbf{H}} \mathbf{S}\right)^{-\mathbf{1}} \mathbf{S}^{\mathbf{H}} \mathbf{Y} \\
\widehat{\mathbf{H}}=\mathbf{S}^{-\mathbf{1}} \mathbf{Y}
\end{gathered}
$$

Where $\mathbf{S}$ is the transmitted signal and $\mathbf{Y}$ is the received signal. After estimating the channel at the pilot positions, these estimates are to be interpolated to other data positions to obtain the channel response for the channel equalization. In [14] the interpolation over frequency and time domain is used for the channel estimation purpose. Pilot based channel estimation techniques of OFDM/OQAM and GFDM differ in one main way i.e. in OFDM/OQAM for estimation, there are two real pilot symbols one to cater the interference and one for the channel estimation. However, in the GFDM complex valued pilot symbols are used which contains the interference cancellation term and intended pilot. For the estimation, both the schemes have same bandwidth efficiency though.

After the LS channel estimation carrier demodulation is carried out through the following equation:

$$
\hat{s}^{\prime}=\sqrt{2}\left(\overrightarrow{\hat{y}}_{\text {real }} \cos \left(2 \pi f_{c} t\right)-\overrightarrow{\hat{y}}_{\text {imag }} \sin \left(2 \pi f_{c} t\right)\right)
$$

Where $\hat{y}$ is the estimated transmitted signal by LS estimation and $\hat{s}^{\prime}$ represents the carrier demodulated signal. A Butterworth filter of order one is then used with:

$$
\omega_{n}=\frac{f_{c}}{f_{s}}
$$

Where $f_{s}$ is the sampling frequency and $f_{c}$ being the carrier frequency. After equalization, carrier demodulation and removing $\mathrm{CP}$, reverse of GFDM is simulated by $\hat{\vec{s}}=A^{H} r$ where $\hat{\vec{s}}$ is the demultiplexed signal and $r$ is the received signal on which is processed through the above sections.

\section{SIMULATION RESULTS}

A Rayleigh fading channel is adopted to evaluate the performance of generalized frequency division multiplexing. The bit error rate (BER) results of the two proposed pilot placement schemes for the channel estimation of GFDM are compared with the previous

\footnotetext{
*Corresponding author: p116006@nu.edu.pk
} 
work done in [7] (Basic GFDM-LS). BER graphs are obtained through Monte Carlo simulation. The parameters used in the simulation of the GFDM are given in the table 2. $E_{b} / N_{0}$ denotes the signal to noise ratio (SNR). Received GFDM signal is detected by Least Squares (LS) frequency domain equalization by first determining the response at the pilot location and then interpolating on the data locations. This way the channel is estimated and then equalized by FDE.

Fig. 5 shows the Fourier transform of the transmitted signal. Since the imaginary part of the data is superimposed on sine wave and the real part of the data is superimposed cosine wave (and then summed up) with the carrier frequency of $10,000 \mathrm{~Hz}$, therefore the Fourier transform shows two peaks at $-10 \mathrm{kHz}$ and $10 \mathrm{kHz}$.

Five different total number of pilot symbols are considered ranges from $1 \%$ of the total symbols in data to $5 \%$ of the data.

In fig. 6 the bit error rate plot of the GFDM with the pilot placement scheme of Method I (i.e. pilots are uniformly arranged on equal intervals with in the data) is shown. The channel estimation is performed using the least squares estimation and then equalization is carried out in the frequency domain. Fig.6a shows the BER plot at $E_{b} / N_{o}=1$ to $E_{b} / N_{o}=8$ for five different lengths of pilot symbols vector. And fig. $6 \mathrm{~b}$ shows the BER plot from $E_{b} / N_{o}=5$ to $E_{b} / N_{o}=30$.

Fig. 7 on the other hand illustrates the bit error rate plot of Method II i.e. the pilots here are clustered in the center of the data. On the receiver end firstly the pilot symbols are extracted from the data the least squares estimation is performed on the pilot points of the data. Then this estimate is interpolated over the complete length of the channel and through this channel equalization is performed in frequency domain (FDE). Same estimation method is used in the processing of fig. 6. Fig. 7 also shows BER for five different total no. of the pilot symbols. Fig $7 \mathrm{a}$ shows the BER plot from $E_{b} / N_{o}=$ 1 to 8 and fig. $7 \mathrm{~b}$ shows the BER plot from $E_{b} / N_{o}=$ 5 to 30 .

Fig. 6 and fig. 7 are simulated using proposed pilot symbol placement techniques for GFDM, which are proved optimum in general in [13]. The results of this paper are compared to the prior works of GFDM BASICLS as in [7], the results show quiet improvement. It is observed that the results of the simulation also satisfies the works of [13], which clearly suggests that the best optimum pilot placement scheme is method 1 .

Table 2. List of parameters in simulation

\begin{tabular}{|c|c|c|c|}
\hline S/No. & Parameter & Notation & Value \\
\hline $\mathbf{1}$ & Digital Modulation & - & QPSK \\
\hline $\mathbf{2}$ & Sub-Carriers & $\mathrm{K}$ & 128 \\
\hline $\mathbf{3}$ & Sub-Symbols & $\mathrm{M}$ & 8 \\
\hline $\mathbf{4}$ & Total no of Samples & $\mathrm{N}$ & 1024 \\
\hline $\mathbf{5}$ & Up Sampling Factor & $U_{F}$ & 10 \\
\hline $\mathbf{6}$ & Cyclic Prefix & $C_{p}$ & 10 \\
\hline $\mathbf{7}$ & Sampling Frequency & $f_{s}$ & $44,100 \mathrm{~Hz}$ \\
\hline $\mathbf{8}$ & Carrier Frequency & $f_{c}$ & $10,000 \mathrm{~Hz}$ \\
\hline
\end{tabular}

*Corresponding author: p116006@nu.edu.pk

\begin{tabular}{|c|c|c|c|}
\hline $\mathbf{9}$ & Block Length & - & 128 \\
\hline $\mathbf{1 0}$ & Pulse Shaping & - & RRC \\
\hline $\mathbf{1 1}$ & Roll off Factor & $\beta$ & 0.5 \\
\hline $\mathbf{1 2}$ & Channel & $\mathrm{w}, \mathrm{h}$ & AWGN, Raleigh \\
\hline $\mathbf{1 3}$ & $\begin{array}{l}\text { Butterworth Filter } \\
\text { normal frequency }\end{array}$ & $\omega_{n}$ & $\begin{array}{l}\frac{f_{c}}{f_{s}}=\frac{10,000}{44,100} \\
=0.227\end{array}$ \\
\hline
\end{tabular}

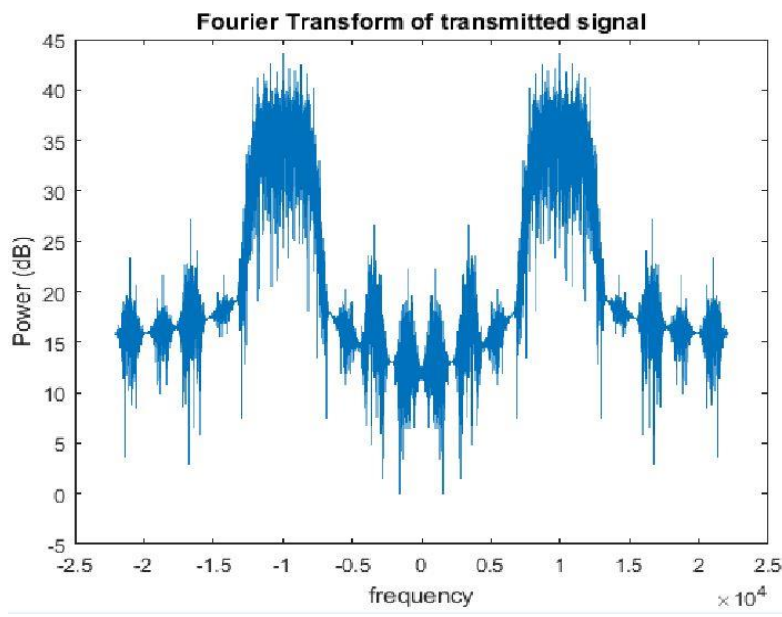

Fig. 5. Fast Fourier transform of transmitted signal

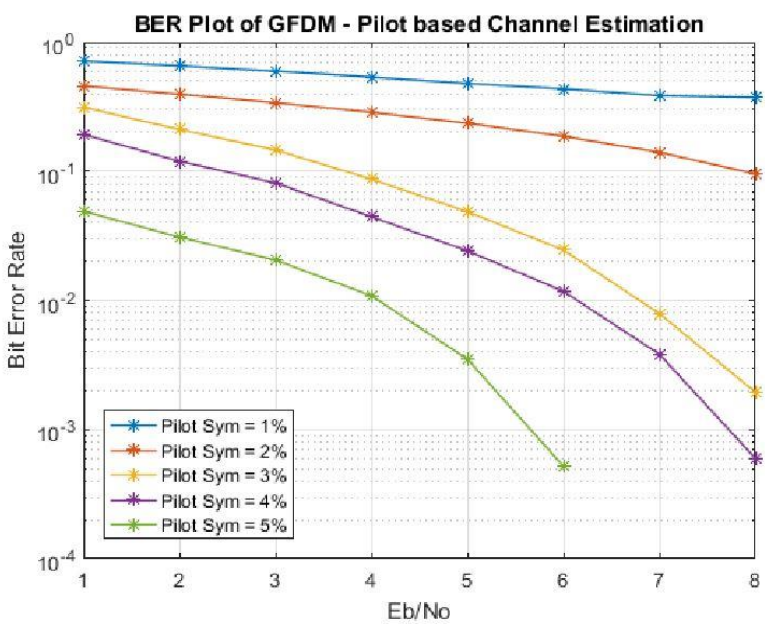

fig. $6 \mathrm{a}$

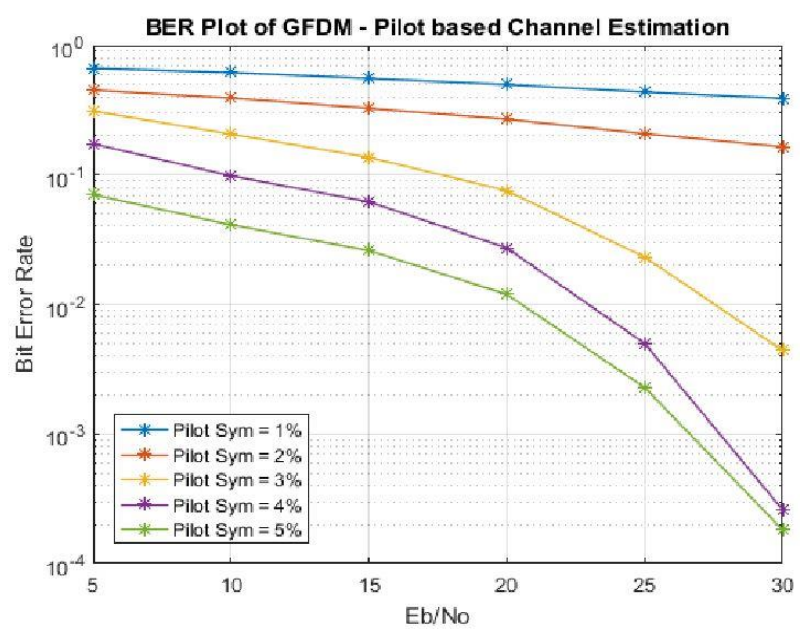

fig. $6 \mathrm{~b}$

Fig. 6. BER plot when Pilot Symbols are arranged on equal intervals in the data (Method I) 


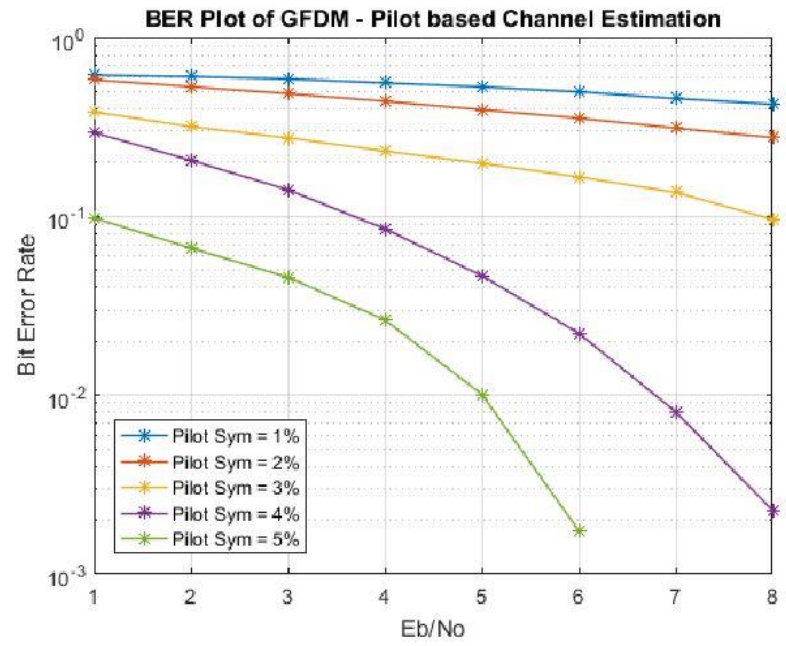

Fig. 7a

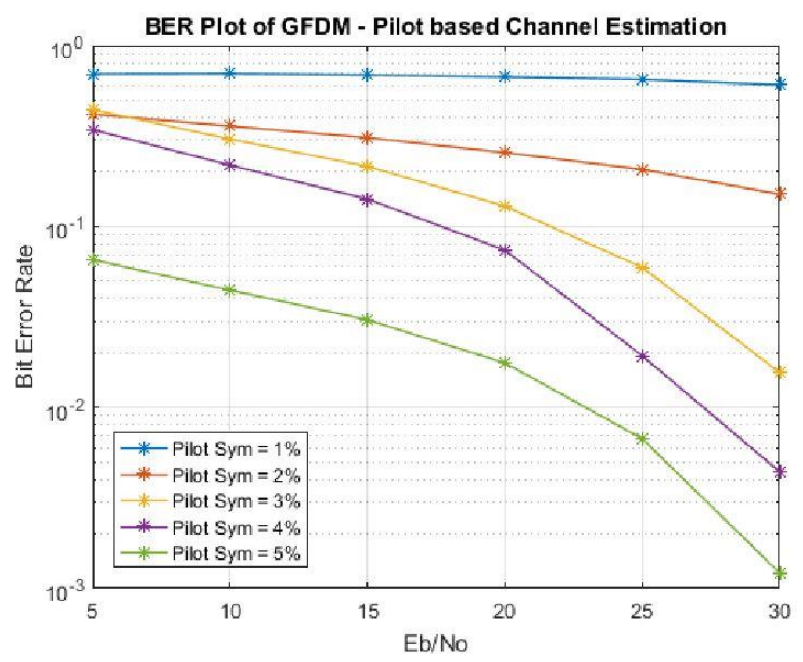

Fig. $7 \mathrm{~b}$

Fig. 7. BER plot when Pilot Symbols are clustered in the center of the data. (Method II)

\section{CONCLUSION}

Two pilot placement techniques are proposed in this paper for channel estimation (one is quasi periodic pilot placement and other is clustering of pilots in the middle of the data) for the non-orthogonal waveform GFDM. In addition, the proposed estimation is frequency domain channel estimation using least squares. Here the bit error rates (BER) for the different lengths of the pilot vector for both proposed pilot schemes are evaluated and compared with each other and with the prior works of GFDM BASIC-LS in [7] with imperfect channel knowledge. From the simulation it is observed that the pilot scheme of method 1 gives the best result if compared to the method 2 and the prior works. In future, more challenging scenarios for the GFDM channel estimation such as highly doubly dispersive channels and CGFDM system proposed in [15], which transmits coded symbols and has the same bandwidth efficiency as GFDM will be explored. In term of exploiting the frequency selectivity, GFDM outperforms the OFDM as in [3].

\section{REFERENCES}

[1] Nicola Michailow, Maximilian Matthé, Ivan Simões Gaspar, Ainoa Navarro Caldevilla, Luciano Leonel Mendes, Andreas Festag, Senior Member, IEEE, and Gerhard Fettweis, "Generalized Frequency Division Multiplexing for 5th Generation Cellular Networks" IEEE transactions on communications, vol. 62, no. 9, september 2014.

[2] G. Fettweis, "The Tactile Internet: Applications and Challenges" IEEE Veh. Technol. Mag., vol. 9, no. 1, pp. 64-70, 2014.

[3] D. Zhang, L. Mendes, M. Matthe, I. Gaspar, N. Michailow, and G. Fettweis, "Expectation Propagation for Near-Optimum Detection of MIMOGFDM Signals" IEEE Trans. Wireless Commun., 2015.

[4] M. Matthe, D. Zhang, and G. Fettweis, "Iterative Detection using MMSE-PIC Demapping for MIMOGFDM Systems" in Proc. of the EW European Wireless Conference (EW'16). IEEE, 2016.

[5] M. Matthe, L. Mendes, N. Michailow, D. Zhang, and G. Fettweis, "Widely Linear Estimation for SpaceTime-Coded GFDM in Low Latency Applications" IEEE Trans. Commun, 2015.

[6] N. Michailow, M. Lentmaier, P. Rost, and G. Fettweis, "Integration of GFDM secondary system in an OFDM primary system" in Future Network and Mobile Summit, 2011.

[7] S. Ehsanfar, M. Matthe, D. Zhang, and G. Fettweis, "Interference-Free Pilots Insertion for MIMOGFDM Channel Estimation" in 2017 IEEE Wireless Communications and Networking Conference (WCNC), May. 2017, pp. 1-6.

[8] N. Michailow, S. Krone, M. Lentmaier, and G. Fettweis, "Bit error rate performance of Generalized Frequency Division Multiplexing" in IEEE Veh. Tech. Conf. (VTC Fall'12), 2012.

[9] R. Heimiller, "Phase shift pulse codes with good periodic correlation properties" IRE Trans. Inform. Theory, vol. IT-7, pp. 254-257, Oct. 1961.

[10] B. Popovic, "Generalized chirp-like polyphase sequences with optimum correlation properties" IEEE Trans. Inform. Theory, vol. 38, pp. 1409, July 1992.

[11] J. C. L. Ng, K. B. Letaief, and R. D. Murch, "Complex optimal sequences with constant magnitude for fast channel estimation initialization" IEEE Trans. Commun, vol. 46, pp. 305-308, Mar. 1998.

[12] S. Ohno and G. B. Giannakis, "Optimal training and redundant precoding for block transmissions with application to wireless OFDM" IEEE Trans. Commun, Nov. 2000, submitted for publication.

[13] Min Dong and Lang Tong, "Optimal Design and Placement of Pilot Symbols for Channel Estimation”, IEEE TRANSACTIONS ON SIGNAL' PROCESSING, VOL. 50, NO. 12, DECEMBER 2002.

[14] JP. Javaudin, D. Lacroix, and A. Rouxel, "Pilot-aided channel estimation for OFDM/OQAM" in IEEE Veh. Tech. Conf. (VTC Spring'03), 2003, pp. 1581-1585.

[15] Mohammad Towliat and Seyyed Mohammad Javad Asgari Tabatabaee, "GFDM Interference Mitigation Without Noise Enhancement" in IEEE Communications Letters (Volume: 22 , Issue: 5 , May 2018)

Corresponding author: p116006@nu.edu.pk 